\title{
Approximation Algorithms for Computing Capacity of Wireless Networks with SINR constraints
}

\author{
Deepti Chafekar*, V. S. Anil Kumar ${ }^{\dagger}$, Madhav V. Marathe, ${ }^{\dagger}$, Srinivasan Parthasarathy ${ }^{\ddagger}$ and \\ Aravind Srinivasan ${ }^{\S}$ \\ *Department of Computer Science, Virginia Tech, Blacksburg, VA 24061, Email: chafekar@vt.edu \\ ${ }^{\dagger}$ Department of Computer Science and Virginia Bioinformatics Institute, Virginia Tech, Blacksburg, VA 24061 \\ Email:\{akumar, mmarathe\}@vbi.vt.edu \\ ‡IBM T.J. Watson Research Center, Hawthorne, NY 10532, Email: spartha@us.ibm.com \\ $\S$ Dept. of Computer Science and Institute for Advanced Computer Studies, \\ University of Maryland, College Park, MD 20742, Email: srin@cs.umd.edu
}

\begin{abstract}
A fundamental problem in wireless networks is to estimate its throughput capacity - given a set of wireless nodes, and a set of connections, what is the maximum rate at which data can be sent on these connections. Most of the research in this direction has focused on either random distributions of points, or has assumed simple graph-based models for wireless interference. In this paper, we study capacity estimation problem using the more general Signal to Interference Plus Noise Ratio (SINR) model for interference, on arbitrary wireless networks. The problem becomes much harder in this setting, because of the non-locality of the SINR model. Recent work by Moscibroda et al. [16], [18] has shown that the throughput in this model can differ from graph based models significantly. We develop polynomial time algorithms to provably approximate the total throughput in this setting.
\end{abstract}

\section{INTRODUCTION}

A fundamental problem in wireless networks is to estimate its throughput capacity - given a set $V$ of wireless nodes, and a set $\mathcal{D}$ of connections, what is the maximum rate at which data can be sent on these connections. Starting with [8], there has been a lot of work on this problem, especially for networks formed by a random distribution of nodes in the unit square. A related, and more practical question is to estimate the capacity of the given network, and develop protocols to utilize the network close to its capacity. This question becomes difficult in wireless networks because of interference, which constrains the set of links that can transmit simultaneously. The algorithmic aspects of network capacity have been studied in a number of papers, such as [2], [9]-[11], [13], [20].

A commonly used approach when designing provable algorithms is to represent the underlying wireless network as a geometric intersection graph. Each node $u \in V$ is associated with a disk of radius range $(u)$, which depends on the transmission power level, $J(u)$ of $u$; a common approximation is to choose range $(u)=\Theta\left((J(u))^{1 / \alpha}\right)$, where $\alpha$ is the path loss exponent, and the signal from node $u$ is assumed to be heard only within this range. This gives us the connectivity graph $G=(V, E)$ obtained by adding links $(u, v)$ to $E$ if $d(u, v) \leq$ range $(u)$. Interference in such a graph is modeled through independence constraints (see e.g., [19]): if a node $u$ transmits, no node in its vicinity can transmit. A number of papers have studied MAC protocols with these geometric models of interference [19], [20]. Intuitively, such graph based models make the algorithmic analysis tractable since they localize the interference effect of a transceiver on others.

While such graph based models give a useful first approximation to understanding wireless networks, they have several limitations. A more realistic model that has been used to study wireless transmission is called the Signal to Interference Plus Noise Ratio (SINR) model [8], [18]: a signal from a transmitter $u$ is successfully received by a receiver at $v$, if the ratio of $u$ 's signal strength at $v$ and the combined interference from other transmitters along with ambient noise exceeds $v$ 's antenna gain. In other words, a set of transmissions $e_{1}=\left(u_{1}, v_{1}\right), \ldots, e_{k}=\left(u_{k}, v_{k}\right)$ can be simultaneously scheduled if for all $e_{i}$,

$$
\frac{J\left(e_{i}\right)}{\ell\left(e_{i}\right)^{\alpha}\left[N_{0}+\sum_{j \neq i} \frac{J\left(e_{j}\right)}{d\left(u_{j}, v_{i}\right)^{\alpha}}\right]} \geq \beta,
$$

where $N_{0}$ denotes the noise density, $\alpha$ denotes the path loss exponent, and $J\left(e_{i}\right)$ denotes the power level with which node $u_{i}$ transmits. Recent work by Moscibroda et al. [16]-[18] has shown that for several problems, this model is significantly different from graph based models. In [16], [18], they show that for the problem of minimizing the scheduling complexity, by choosing appropriate transmission power levels, SINR models allow for much shorter schedules. In [17], they show that the throughput capacity under an SINR model is different from that under a graph based model. The non-locality of this model makes its analysis challenging.

In this paper we consider the problem of characterizing the achievable rates for arbitrary multi-hop wireless networks with SINR constraints. Given a set of nodes $V$, a set of source-destination pairs $\mathcal{D}=\left\{\left(s_{1}, t_{1}\right), \ldots,\left(s_{k}, t_{k}\right)\right\}$, and a power level $J(e)$ for transmission on edge $e$, the throughput maximization problem with SINR constraints (TM-SINR) consists of (i) choosing routes for the connections, (ii) choosing flow rates on the routes, and (iii) scheduling the packets 
at each time such that the SINR constraints are satisfied for all simultaneous transmissions, and that the total throughput capacity is maximized. Note that the TM-SINR problem does not involve power control, i.e., the power levels $J(e)$ for each edge are fixed and given as part of the input. The SINR constraints make the throughput optimization problem nonconvex. Further the link scheduling problem with SINR constraints has shown to be NP-complete in [7]. Since scheduling is also an integral component of our problem, it is reasonable to conjecture that the throughput maximization problem is also NP-complete. We focus on developing rigorous polynomial time methods with provable performance guarantees.

In reality, the link capacities depend on the SINR [1], therby making this problem very complex. We simplify this by using the Additive White Gaussian Noise (AWGN) model for specifying the link capacities [4]. In this model the capacity, $\operatorname{cap}(e)$, of a link $e$ having length $\ell(e)$ and transmitting at power level $J(e)$ is given by

$$
\operatorname{cap}(e)=W \log _{2}\left(1+\frac{J(e)}{\ell(e)^{\alpha} N_{0} W}\right),
$$

where $W$ is the bandwidth, and $N_{0}$ and $\alpha$ are as defined earlier. In the absence of interference, the above equation provides a theoretical upper bound on the link capacity. However, the maximum throughput problem with SINR constraints remains non-trivial even under the AWGN model, and we only study this here.

\section{OVERVIEW OF RESULTS}

We study the TM-SINR problem in wireless networks from a theoretical perspective, and take the first steps towards developing efficient algorithms for this problem. The main contributions of our work are summarized below.

- We compare the SINR and graph based models for the same instance, with the same fixed power levels, and observe that the throughput capacity can be significantly different in these two models. When the power level for all the edges is the same, we show that there are instances in which the throughput capacity that can be achieved in the SINR model is significantly higher than that in the graph based model. For the case of linear power levels (where $J(e) \propto \ell(e)^{\alpha}$, for each edge $e$ ), we show that there are instances in which the throughput capacity in the SINR model can be much lower than in the corresponding graph based model with the same power levels. In contrast, the results of [16]-[18] show that by choosing suitable power levels, a much higher throughput capacity is possible in SINR models than in graph based models. Since all these models of interference are approximations of the real phenomenon, this suggests greater care is needed in inferring any properties of the system based on such an analysis.

- We develop a linear programming based approach to approximate the maximum throughput rate vector in the case of SINR constraints. For the case of uniform power levels (when all nodes have the same power level $J$ ), we develop a polynomial time approximation algorithm that provides a feasible rate vector whose total throughput is at least $\Omega\left(r_{o p t} / \log \Delta\right)$, where $r_{o p t}$ is the maximum possible throughput for this instance and $\Delta$ is defined as $\Delta=\max _{u, v \in V} d(u, v) / \min _{u^{\prime}, v^{\prime} \in V, u^{\prime} \neq v^{\prime}} d\left(u^{\prime}, v^{\prime}\right)$. This gives us an $O(\log \Delta)$ approximation to the total throughput. Our approximation bound is a worst case guarantee that holds for every instance.

- Next, we consider the case of non-uniform power levels, in which the power levels on different edges could be different. We extend our method to obtain an $O(\log \Delta \log \Gamma)$ approximation to the total throughput, where $\Gamma$ is the ratio between maximum and minimum power levels used.

- We consider a special case of non-uniform power levels, called linear power level choice, where the power level on each edge $e$ is $J(e)=c_{1} \ell(e)^{\alpha}$ for a constant $c_{1}$. In this case, we improve the $O\left((\log \Delta)^{2}\right)$ approximation to just $O(\log \Delta)$.

For technical reasons, the $O(\log \Delta)$ bound is only relative to the optimum rate possible by using slightly smaller power levels - this is explained formally in Section VI. Our algorithm builds upon the recent work of [6], [16], [18] on scheduling with SINR constraints, and the LP based approaches of [10], [13] for estimating the capacity for graph-based interference models.

\section{RELATED WORK}

There has been significant work on understanding the capacity of random networks formed by nodes distributed randomly in the plane, using both graph based and SINR models (see, e.g., [3], [8], [12]), and for other variants of such distributions. However, these results do not directly help in understanding the capacity of arbitrary networks, which is the focus of our paper. The throughput maximization problem for graph based models is formally studied and proven to be NP-hard by Jain et. al. [9], who use a linear programming approach to characterize the capacity of the network and to perform routing. They model interference constraints as a conflict graph and provide upper and lower bounds for optimal throughput. As mentioned in [5], the methods discussed in [9] tend to have an exponential complexity and no performance guaranteed polynomial time approximation algorithm is proposed. Toumpis et. al. [21] provide a mathematical framework for determining the capacity region of an ad-hoc network, which captures the effects of power control, spatial reuse and successive interference cancellation on the capacity region. However, their results do not give worst case approximation guarantees.

Kodialam et. al. [10] study the problem of determining achievable rates for multi-hop wireless, along with joint routing and scheduling constraints in graph based models. Their approach provides necessary and sufficient conditions for link flows and leads to a polynomial time approximation algorithm for this problem. However, they only consider primary interference in their model, which is very restrictive. 
Lin et. al. [14], [15] study the joint problem of rate allocation and scheduling using a dual optimization based approach to decompose the problem as rate control and scheduling problem. Their technique provides an optimal solution that maximizes the throughput and provides a stable and fair schedule considering the primary interference model. Although some of these approximation bounds have been improved in recent work by Buragohain et. al. [5], it is not intuitive to extend these techniques for the SINR interference model.

Some of the key algorithmic results on the SINR model are studied in [6], [16]-[18]. Moscibroda et al. [16]-[18] study the problem of scheduling edges with SINR constraints to ensure that some property (e.g., connectivity) is satisfied by the edges that are chosen. They show that by suitable power control, the solutions in the SINR model are much more efficient than those in graph based models. Chafekar et al. [6] develop approximation algorithms for packet scheduling to minimize end-to-end delays with SINR constraints.

Our work is closely related to Kumar et. al. [6], [13]. The work by [13] provides a constant approximation algorithm for the throughput maximization problem along with joint scheduling and routing. The interference model considered is graph-based and their approach is generic enough to accommodate the case of uniform and non-uniform power levels. They further derive linear necessary and sufficient conditions that lead to a constant factor approximation to the throughput capacity. However, the framework presented in [13] cannot be easily extended to the SINR interference model. In this work, by combining some of the techniques from [6], [13], we study the throughput maximization problem along with joint routing and scheduling for the SINR interference constraints.

\section{PRELIMINARIES}

We consider the input instance of the TM-SINR problem to be specified as $\mathcal{I}=(V, E, \mathcal{D}, \bar{J})$, where (i) $V$ denotes a set of transceivers, henceforth referred to as nodes, which are located on the plane, (ii) $E \subseteq V \times V$ denote the set of possible links (also referred to as edges), on which transmissions can occur, (iii) $\mathcal{D}$ is a set of connections, with the $i$ th connection from node $s_{i}$ to node $t_{i}$, and (iv) $\bar{J}=(J(e): e=(u, v) \in E)$ specifies the vector of power transmission levels on edges. For $u, v \in V$, let $d(u, v)$ denote the Euclidean distance between these nodes; for $e=(u, v) \in E$, let $\ell(e)=d(u, v)$. Following standard graph theory notation, let $N_{\text {out }}(u)$ and $N_{\text {in }}(u)$ be the sets of outgoing and incoming edges for node $u$, respectively. Let $\Delta=\max _{e \in V}\{\ell(e)\} / \min _{e^{\prime} \in E}\left\{\ell\left(e^{\prime}\right)\right\} ; \log \Delta$ is also called the "length diversity" [7]. All the logarithms are to the base two. Without loss of generality, we assume that $\min _{e \in E}\{\ell(e)\}=1$. We define $B_{i}=\{e \in E$ : $\left.\ell(e) \in\left[2^{i}, 2^{i+1}\right)\right\}$, for $i \in\{0, \ldots,(\log \Delta-1)\}$. Also, let $\Gamma=\frac{\max _{e} J(e)}{\min _{e^{\prime}} J\left(e^{\prime}\right)}$.

Note that for edge $e \in E$, its power level $J(e)$ is given, and so our assumption of the AWGN model (equation 1) implies that its capacity $\operatorname{cap}(e)$ is also fixed.

\section{A. Interference Model}

We use the SINR model of interference as described in [6], [18]. In this setting, a given set $E^{\prime}=\left\{e_{i}=\left(u_{i}, v_{i}\right)\right.$ : $i=1, \ldots, k\}$ of links can simultaneously communicate successfully if for each $e_{i} \in E^{\prime}$, we have

$$
\operatorname{SINR}\left(v_{i}\right)=\frac{J\left(e_{i}\right)}{\ell\left(e_{i}\right)^{\alpha}\left[N_{0}+I_{r}\left(v_{i}, E^{\prime}\right)\right]} \geq \beta,
$$

where (i) $N_{0}$ denotes the ambient noise density, which is a constant, (ii) $\beta$ is a constant, related to the antenna properties, (iii) $\alpha$ denotes the path-loss exponent, which we assume to be greater then 2 , and (iv) $I_{r}\left(v_{i}, E^{\prime}\right)=\sum_{e_{j} \neq e_{i}} \frac{J\left(e^{\prime}\right)}{d\left(u_{j}, v_{i}\right)^{\alpha}}$ denotes the interference at receiver $v_{i}$ due to all other transmissions - we will simply denote this as $I_{r}\left(v_{i}\right)$ if the set $E^{\prime}$ is clear from the context.

Note that in this model, for any edge $e=(u, v) \in E$, we need $J(e) \geq \beta N_{0} \ell(e)^{\alpha}$ for the transmission on this edge to be feasible, even in the absence of any other interference. We will assume that for an instance $\mathcal{I}=(V, E, \mathcal{D}, \bar{J})$ of TM-SINR, we have $J(e) \geq \beta N_{0} \ell(e)^{\alpha}$ for all $e \in E$.

\section{B. Link rates and feasible end-to-end schedules}

We assume that the time is divided into uniform slots, each of duration $\tau$ and the system operates in a synchronous mode. Let $\mathcal{D}=\{1, \ldots, k\}$ denote a set of connections, with $s_{i}$ and $t_{i}$ denoting the source and destination respectively, for connection $i$. Let $f_{i}(e)$ denote the mean flow rate on link $e$ for the $i$ th connection, and let $f(e)=\sum_{i} f_{i}(e)$ denote the total link flow. We let $x(e)=f(e) / \operatorname{cap}(e)$ denote the link utilization - this denotes the fraction of time link $e$ is used. The vectors $\bar{f}$ and $\bar{x}$ are called the flow vector and link utilization vector respectively. An end-to-end schedule $\mathcal{S}$ describes the specific times at which packets are transmitted over the links of the network. For schedule $\mathcal{S}$, let $X(e, t)$ be an indicator variable that is 1 if the link $e$ is used at time $t$. We say that $\mathcal{S}$ is valid if the SINR constraints are satisfied at all the receivers at every time $t$. We say that $\mathcal{S}$ feasibly schedules the link utilization vector $\bar{x}$ if we have $\lim _{T \rightarrow \infty} \sum_{t \leq T} \frac{X(e, t)}{T}=x(e)$ for each edge $e$ - in this case, we say that $\mathcal{S}$ corresponds to the utilization vector $\bar{x}$. The rate region $\mathcal{X}(\mathcal{I})$ is the space of all utilization vectors $\bar{x}$ for the instance $\mathcal{I}$ of TM-SINR that can be scheduled feasibly.

Let $r_{i}$ denote the end-to-end rate on the $i$ th connection in bits per second, resulting from the flow vector $\bar{f}$. In this paper we are interested in maximizing the total end-to-end rate $\sum_{i} r_{i}$. For an instance $\mathcal{I}=(V, E, \mathcal{D}, \bar{J})$ of TM-SINR, let $r_{\text {opt }}(\mathcal{I})$ denote the maximum possible total throughput rate that is feasible for this instance. We say that a utilization vector $\bar{x} \in \mathcal{X}(\mathcal{I})$ is a $\gamma$-approximation to the throughput maximization problem if the resulting total rate achieved is at least $\gamma \cdot r_{\text {opt }}(\mathcal{I})$; we say that an algorithm is a $\gamma$ approximation algorithm, if for any instance $\mathcal{I}$ of TM-SINR, it provably produces such a $\gamma$-approximate solution $\bar{x} \in \mathcal{X}(\mathcal{I})$ in polynomial time, for any instance $\mathcal{I}$ of TM-SINR- note that this is a worst case approximation result. 


\section{Congestion Measure}

Following [6], we define a notion of congestion $C$, that will play a key role in our algorithm. For $e=(u, v) \in E$, let

$C(e)=\left\{e^{\prime}=\left(u^{\prime}, v^{\prime}\right) \in E: a \cdot \ell\left(u^{\prime}, v^{\prime}\right) \geq d\left(u, u^{\prime}\right) \wedge \ell\left(e^{\prime}\right) \geq \ell(e)\right\}$,

and let $C=\max _{e \in E}|C(e)|$. Here, $a$ is a constant such that $a \geq 4 \sqrt[\alpha]{\frac{48 \beta(1+\epsilon)}{\epsilon(\alpha-2)}}, \epsilon$ is a small positive constant and $\alpha>2$ [18]. The significance of the congestion $C$ is that it provides a lower bound on the number of feasible simultaneous transmissions [6], which we use to approximate $r_{\text {opt }}$.

\section{SINR VS GRAPH BASED MODELS}

In this section, we compare the SINR and graph models in the context of the throughput maximization problem. Given an instance $\mathcal{I}=(V, E, \mathcal{D}, \bar{J})$ of TM-SINR, we follow the approach of [18] in constructing an "equivalent" connectivity graph $G=\left(V, E_{g m}\right)$ and a resulting instance $\mathcal{I}_{g m}$ in a graph based model in the following manner. Recall the notation from Section IV. In the rest of this section, we will consider instances $\mathcal{I}$ of TM-SINR in which every node $u \in V$ uses a fixed power level $J(u)=J(e)$ for every incident link $e=(u, v) \in E$. We associate a transmission range of $r(u)=\left(J(u) / c_{1}\right)^{1 / \alpha}$ with every node $u \in V$, giving rise to a disk graph $G=\left(V, E_{g m}\right)$ with $(u, v) \in E_{g m}$ if $d(u, v) \leq r(u)$. This is a directed graph in general, if nodes have non-uniform transmission ranges. The corresponding instance $\mathcal{I}_{g m}$ consists of this graph $G$ along with the same set $\mathcal{D}$ of connections, as in $\mathcal{I}$. Note that the set of edges on which transmissions can happen is the same in both models. For every edge $e \in E_{g m}$, we use the same expression for $\operatorname{cap}(e)$, the capacity of edge $e$ as in $\mathcal{I}$, since this comes from the AWGN model. What is different is the interference - we can now use any graph based interference model to specify the set $I_{g m}(e)$ of edges that interfere with $e$ - for concreteness, we use the distance-2 matching model [13], which defines $I_{g m}(e)=\left\{e^{\prime}=\left(u^{\prime}, v^{\prime}\right): d_{G}\left(\{u, v\},\left\{u^{\prime}, v^{\prime}\right\}\right) \leq 1\right\}$, where $d_{G}()$ defines the distance between two sets in the graph $G$. A schedule is valid in the graph-based model, if at any time, no edge $e$ is simultaneously scheduled along with some edge $e^{\prime} \in I(e)$. Let $r_{o p t}^{g m}\left(\mathcal{I}_{g m}\right)$ denote optimum throughput rate possible for this instance in the graph based model.

We show the following results in this section.

- If the instance $\mathcal{I}$ of TM-SINR has uniform power levels, the ratio $r_{o p t}(\mathcal{I}) / r_{o p t}^{g m}\left(\mathcal{I}_{g m}\right)$ can be arbitrarily large, i.e., the corresponding graph based model underestimates the throughput capacity significantly.

- In contrast, when the power levels in the instance $\mathcal{I}$ of TM-SINR are linear, we show that the ratio $r_{\text {opt }}(\mathcal{I}) / r_{\text {opt }}^{g m}\left(\mathcal{I}_{g m}\right)$ can be arbitrarily small.

The above results show that if the power levels are fixed, the total throughput in both the models is very different - this is in contrast to the results of [16], [18], which show that by choosing appropriate power levels, a much higher throughput is possible in the SINR model for the same instance.

\section{A. Uniform power levels}

We construct the following instance $\mathcal{I}=(V, E, \mathcal{D}, J)$ of TM-SINR, with uniform power level $J$ for all transmissions. Let $R=\left(J / c_{1}\right)^{1 / \alpha}$ be the corresponding transmission range in the corresponding graph model, as discussed earlier; we assume that $R$ is a large integer. Let $V=\left\{v_{0}\right\} \cup_{i=1}^{n}\left\{v_{i}, w_{i}\right\}$ be a set of nodes, which are placed in the following manner. Imagine a circle of radius $R / 2$ centered at node $v_{0}$, and the nodes $v_{1}, \ldots, v_{n}$ are uniformly placed on the circumference of this circle at a spacing of $\Theta(\sqrt{R})$, so that $n=\Theta(\sqrt{R})$. Each $w_{i}$ is at a unit distance from $v_{i}$, for $i=1, \ldots, n$. Let the connections in $\mathcal{D}$ in the instance $\mathcal{I}$ be all the pairs $e_{i}=$ $\left(v_{i}, w_{i}\right)$, for all $i$. Let $c a p=\operatorname{cap}\left(e_{i}\right)$ denote the capacity of any link $e_{i}$ in bits/sec; note that this is the same for every edge $e_{i}$ in this setting. For simplicity, we ignore the ambient noise, i.e., assume $N_{0}=0$. It is easy to extend these results to take the noise into account.

Lemma 1: For the instance $\mathcal{I}$ of TM-SINR and the corresponding graph-based instance $\mathcal{I}_{g m}$ described above, we have $r_{\text {opt }}(\mathcal{I}) / r_{\text {opt }}^{g m}\left(\mathcal{I}_{g m}\right)=\Omega(\operatorname{cap} \cdot \sqrt{R})$, assuming $\beta \leq$ $c_{3} \cdot R^{(\alpha-1) / 2}$, for a constant $c_{3}$.

Proof: Observe that for all $i \neq j, \sqrt{R} \leq d\left(v_{i}, v_{j}\right) \leq R$. Therefore, $I_{g m}\left(e_{i}\right)=\left\{e_{j}: j \neq i\right\}$, which implies that at any time, at most one edge $e_{i}$ can be scheduled in the graph-based model in the instance $\mathcal{I}_{g m}$. This implies that $r_{o p t}^{g m}\left(\mathcal{I}_{g m}\right)=$ $\Theta($ cap $)$ bits/sec.

Next, consider the SINR model for the instance $\mathcal{I}$ of TMSINR. Suppose all the edges $e_{i}$ are scheduled simultaneously - the SINR ratio at any receiver $v_{i}$ in this case is

$\frac{J}{\ell\left(e_{i}\right)^{\alpha}\left[\sum_{j \neq i} J / d\left(u_{j}, v_{i}\right)^{\alpha}\right]} \geq \frac{c_{1} R^{\alpha}}{\left[\sum_{j \neq i} c_{1} R^{\alpha} /\left(c_{2} \cdot \sqrt{R}\right)^{\alpha}\right]} \geq \beta$,

where the first inequality follows from the fact that $J=c_{1}$. $R^{\alpha}$, and $d\left(u_{j}, v_{i}\right)=\Omega(\sqrt{R})$ for this instance, and the second inequality follows if $\beta \leq c_{3} \cdot R^{(\alpha-1) / 2}$ for a constant $c_{3}$. This implies that all the edges $e_{i}$ can be scheduled simultaneously in the SINR model, leading to $r_{\text {opt }}(\mathcal{I})=\Theta(\operatorname{cap} \cdot \sqrt{R})$, and so the lemma follows.

\section{B. Linear Power Levels}

We now construct an instance $\mathcal{I}=(V, E, \mathcal{D}, \bar{J})$ of TMSINR with uniform power levels, i.e., for each $e \in E, J(e)=$ $c_{1} \ell(e)^{\alpha}$. The set $V=\cup_{i=1}^{n}\left\{u_{i}, v_{i}\right\}$ has $2 n$ nodes, which are located on a line in the order $u_{1}, v_{1}, u_{2}, v_{2}, \ldots, u_{n}, v_{n}$. For all $i=1, \ldots, n$, we have $d\left(u_{i}, v_{i}\right)=R_{i}=2^{i}$, and for all $i=1, \ldots, n-1$, we have $d\left(v_{i}, u_{i+1}\right)=2^{i+2}$. The set $E=\left\{e_{i}=\left(u_{i}, v_{i}\right): i=1, \ldots, n\right\}$ will be the only edges used for transmission, with $J\left(e_{i}\right)=c_{1} R_{i}^{\alpha}$, for each $i$. All the connections in $\mathcal{D}$ in this instance are the pairs $e_{i}=\left(u_{i}, v_{i}\right)$, for $i=1, \ldots, n$. Because of our AWGN model for the link capacities, as discussed in Equation 1, it follows that for all $e_{i} \in E, \operatorname{cap}\left(e_{i}\right)=\operatorname{cap}$ is a fixed value. Each node $u_{i}$ has only one incident edge in the set $E$, so for the graph based model, we set $r\left(u_{i}\right)=R_{i}$, as discussed earlier. Therefore, for 
the corresponding graph based instance $\mathcal{I}_{g m}$ the connectivity graph $G=\left(V, E_{g m}\right)$ has $E_{g m}=\left\{e_{i}: i=1, \ldots, n\right\}$.

Lemma 2: For the instance $\mathcal{I}$ and the corresponding graph-based instance $\mathcal{I}_{g m}$ described above, we have $r_{\text {opt }}^{g m}\left(\mathcal{I}_{\text {gm }}\right) / r_{\text {opt }}(\mathcal{I})=\Theta(n)$.

Proof: First, observe that for the graph-based interference in the instance $\mathcal{I}_{g m}$, we have $I_{g m}\left(e_{i}\right)=\phi$ for each $e_{i} \in E_{g m}$. Therefore, the edges $e_{i}$ do not interfere with each other and all these edges can transmit simultaneously in this model, leading to a throughput capacity of $\Omega(n \cdot c a p)$.

Next, consider the SINR model. For simplicity, we ignore the noise density $N_{0}$, though it can be easily incorporated. Let $E^{\prime}$ be any subset of these edges that can transmit simultaneously, and let $e_{i}$ be the shortest among them. For all $e_{j} \in E^{\prime}, e_{j} \neq e_{i}$, we have $d\left(u_{j}, v_{i}\right) \leq \sum_{k=i}^{j-1}\left(2^{k+2}+\right.$ $\left.2^{k+1}\right) \leq c_{2} 2^{j}=c_{3} R_{j}$, for constants $c_{2}, c_{3}$. In order for these transmissions to be feasible in the SINR model, we must have

$$
\frac{J\left(e_{i}\right)}{\ell\left(e_{i}\right)^{\alpha}\left[\sum_{e_{j} \in E^{\prime}, e_{j} \neq e_{i}} \frac{J\left(e_{j}\right)}{d\left(u_{j}, v_{i}\right)^{\alpha}}\right]} \geq \beta,
$$

where the LHS is the SINR ratio at $v_{i}$. Rearranging, and using the fact that $d\left(u_{j}, v_{i}\right) \leq c_{3} R_{j}$ for each $e_{j} \in E^{\prime}$, we have $\left|E^{\prime}\right|$ is $O(1 / \beta)$, which is a constant. This implies $r_{\text {opt }}(\mathcal{I})=$ $O(\operatorname{cap} / \beta)$, and so the lemma follows.

\section{Throughrut Maximization for Uniform Power LEVELS}

\section{A. Problem Formulation}

In this Section we formulate the TM-SINR problem for uniform power levels. We consider input instances of TMSINR specified as $\mathcal{I}=(V, E, \mathcal{D}, J)$ with a uniform power level of $J(e)=J$ for every edge $e \in E$. Recall the notation from section IV. It is easy to see that the exact formulation of the TM-SINR problem is non-convex. We develop a linear programming relaxation of this problem by combining the approaches of [6], [13] - we show that both necessary and sufficient conditions can be derived for the feasible rate region by considering the total link utilization in the edges in the set $C(e)$ for any edge $e$. Our formulation for instance $\mathcal{I}$ described below is denoted by $\mathcal{P}(\lambda, \mathcal{I})$, where $\lambda$ is a parameter.

$$
\begin{array}{r}
\max \sum_{i \in \mathcal{D}} r_{i} \text { subject to: } \\
\forall i \in \mathcal{D}, r_{i}=\sum_{e \in N_{\text {out }}\left(s_{i}\right)} f_{i}(e)-\sum_{e \in N_{\text {in }}\left(s_{i}\right)} f_{i}(e) \\
\forall e \in E, x(e)=\sum_{i \in \mathcal{D}} f_{i}(e) / \operatorname{cap}(e) \\
\forall i \in \mathcal{D}, \forall u \neq s_{i}, t_{i}, \sum_{e \in N_{\text {out }}(u)} f_{i}(e)=\sum_{e \in N_{\text {in }}(u)} f_{i}(e) \\
\forall e \in E, \sum_{e^{\prime} \in C(e)} x\left(e^{\prime}\right) \leq \lambda
\end{array}
$$

In the above formulation, constraints (2) define the total rate $r_{i}$ for each connection, constraints (3) define the link utilization $x(e)$ for each link $e$, constraints (4) ensure flow conservation, and constraints (5) are relaxed congestion constraints - these are the key constraints that allow us to use this program to derive upper and lower bounds on the optimum rate. The program $\mathcal{P}(\lambda, \mathcal{I})$ has polynomial size and can be solved in polynomial time.

In the subsequent sections, we show that the optimum utilization vector satisfies $\mathcal{P}(\lambda, \mathcal{I})$ for some constant value of $\lambda$. We then show that scaling the constraints down allows us to schedule the flow feasibly.

\section{B. Link-Flow Scheduling: Necessary Conditions}

The following lemma shows that $\mathcal{P}(\lambda, \mathcal{I})$ gives an upper bound on $r_{\text {opt }}(\mathcal{I})$ for a suitable choice of $\lambda$.

Lemma 3: Let $\mathcal{I}=(V, E, \mathcal{D}, J)$ be an instance of the TMSINR problem with uniform power level $J$, and let $\bar{x} \in \mathcal{X}(\mathcal{I})$ be any feasible link utilization vector. Then, $\bar{x}$ is a feasible solution to the program $\mathcal{P}\left(\lambda_{0}, \mathcal{I}\right)$, where $\lambda_{0}=\frac{(2 a+1)^{\alpha}}{\beta}+1$, and $a$ is the constant defined in Section IV-C.

Proof: Since $\mathcal{X}(\mathcal{I})$ denotes the set of all feasible utilization vectors for the instance $\mathcal{I}$ of TM-SINR, it is clear that $\bar{x}$, and the associated flow rate vector $\bar{f}$, must satisfy all the constraints of $\mathcal{P}\left(\lambda_{0}, \mathcal{I}\right)$, except possibly the constraints (5). We now argue that the constraints (5) hold for this choice of $\lambda_{0}$.

Since the link utilization vector $\bar{x}$ is feasible, there exists a stable schedule $\mathcal{S}$ which achieves the link rates specified by $\bar{x}$. Recall the notation $X(e, t)$ from Section IV. Let $E_{t}=\{e$ : $X(e, t)=1\}$ denote the set of links that transmit at time $t$ in this schedule.

We now focus on any edge $e=(u, v) \in E_{t}$. Let $A_{t}=$ $E_{t} \cap C(e)=\left\{e_{j}=\left(u_{j}, v_{j}\right) \in C(e): j=1, \ldots, c\right\}$ be a set of $c$ links in $C(e)$ that are scheduled simultaneously at time $t$. We argue below that $c$ must be bounded by a constant. Let the links in the set $A_{t}$ be numbered in non-decreasing order of their lengths, so that $\ell\left(u_{1}, v_{1}\right) \leq \ell\left(u_{2}, v_{2}\right) \leq \ldots \leq \ell\left(u_{c}, v_{c}\right)$. For simultaneously successful transmission of these links, the SINR at each node $v_{j}$, and in particular, at node $v_{c}$, needs to be at least $\beta$.

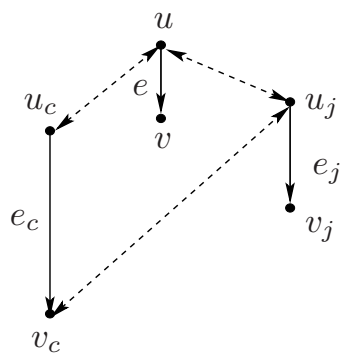

Fig. 1. For a given link $e=(u, v)$ and set $A_{t}, d\left(u_{j}, v_{c}\right) \leq(2 a+$ 1) $d\left(u_{c}, v_{c}\right)$, where $e_{c}, e_{j} \in C(e)$ and $e_{c}$ is the link with longest length in set $A_{t}$.

Consider any $e_{j} \in A_{t}, e_{j} \neq e_{c}$ (cf. Figure 1 ). We have

$$
\begin{aligned}
d\left(u_{j}, v_{c}\right) & \leq d\left(u, u_{j}\right)+d\left(u, u_{c}\right)+d\left(u_{c}, v_{c}\right) \\
& \leq 2 a d(u, v)+d\left(u_{c}, v_{c}\right) \\
& \leq(2 a+1) d\left(u_{c}, v_{c}\right),
\end{aligned}
$$


where the first inequality follows from triangle inequality, and the last two inequalities follow from the definition of $C(e)$, which implies that for any $e^{\prime}=\left(u^{\prime}, v^{\prime}\right) \in C(e)$, we must have $d\left(u, u^{\prime}\right) \leq a \cdot \ell\left(e^{\prime}\right)$ and $\ell(e) \leq \ell\left(e^{\prime}\right)$.

The interference experienced at $v_{c}$ due to all transmitting links in $A_{t}-\left\{e_{c}\right\}$ is

$$
I_{r}\left(v_{c}\right)=\sum_{e_{j}=\left(u_{j}, v_{j}\right) \in A_{t}, j \neq c .} \frac{J}{d\left(u_{j}, v_{c}\right)^{\alpha}} .
$$

Therefore, in order to satisfy the SINR constraint at node $v_{c}$ we need,

$$
\frac{J / d\left(u_{c}, v_{c}\right)^{\alpha}}{\left[N_{0}+\sum_{e_{j} \in A_{t}, j \neq c} J / d\left(u_{j}, v_{c}\right)^{\alpha}\right]} \geq \beta .
$$

Rearranging, we have

$$
\begin{aligned}
\frac{J}{d\left(u_{c}, v_{c}\right)^{\alpha}} & \geq \beta\left[N_{0}+\sum_{e_{j} \in A_{t}, j \neq c} \frac{J}{d\left(u_{j}, v_{c}\right)^{\alpha}}\right] \\
& \geq \beta N_{0}+\frac{\beta J(c-1)}{(2 a+1)^{\alpha} d\left(u_{c}, v_{c}\right)^{\alpha}} .
\end{aligned}
$$

This in turn implies $c \leq \frac{(2 a+1)^{\alpha}}{\beta}+1$, and therefore, we have

$$
\forall e \in E, \forall t, \sum_{e^{\prime} \in C(e) \cap E_{t}} X\left(e^{\prime}, t\right) \leq \lambda_{0},
$$

which implies for any $T$

$$
\forall e \in E, \sum_{e^{\prime} \in C(e)} \sum_{t \leq T} X\left(e^{\prime}, t\right) \leq T \lambda_{0} .
$$

Dividing both sides of (6) by $T$, the lemma follows from the definition of $x(e)$ in Section IV-B.

\section{Link-Flow Scheduling: sufficient conditions}

In this section, we show that the program $\mathcal{P}(\lambda, \mathcal{I}=$ $(V, E, \mathcal{D}, J))$ can be used to derive sufficient conditions for link flow stability for the instance $\mathcal{I}$ of TM-SINR, for a suitable value of the parameter $\lambda$. This requires showing that a solution $\bar{x}$ to this program can be scheduled feasibly, under suitable conditions on $\lambda$ and $J$. We describe algorithm FrameSchedule for constructing a feasible schedule below.

We assume that time is divided into sufficiently large frames of length $w$, and that $x(e) w$ is an integral for all $e \in E$. Recall the definitions of $\Delta$ and the sets $B_{i}$ from Section IV. We further subdivide each frame $W$ into $\log \Delta$ sub-frames $W_{i}$, each of length $w / \log \Delta$, which is assumed to be integral. Algorithm FrameSchedule constructs a periodic schedule $\mathcal{S}$ by repeating a schedule $\mathcal{S}_{W}$ for every frame $W$. Within each sub-frame $W_{i}$, the algorithm considers only the edges from the set $B_{i}$, and assigns $x(e) \cdot w$ slots for each edge $e \in B_{i}$ by a greedy coloring step.

For the above algorithm to be stable, we need to find conditions under which, step 9 of the algorithm would be successful. The following lemma proves that for a suitable value of $\lambda$, the algorithm is indeed successful.

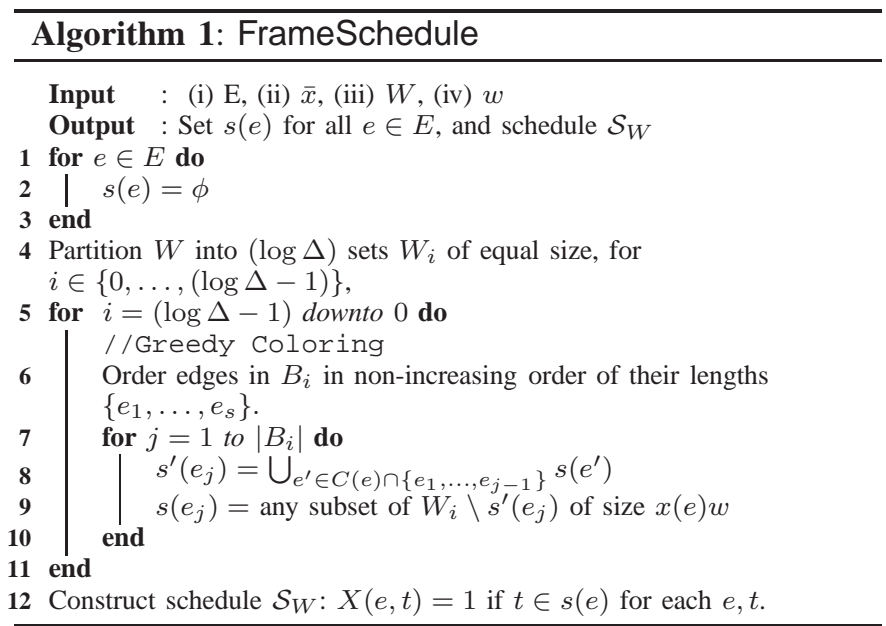

Lemma 4: Algorithm FrameSchedule correctly assigns $|s(e)|=x(e) w$ slots for each edge $e$, if the link utilization vector $\bar{x}$ is any feasible solution to the program $\mathcal{P}\left(\frac{1}{(1+\log \Delta)}, \mathcal{I}\right)$.

Proof: Suppose step 9 of Algorithm FrameSchedule fails for some edge $e_{j} \in B_{i}$. Then, we must have

$$
\sum_{e^{\prime} \in C\left(e_{j}\right) \cap B_{i}}\left|s\left(e^{\prime}\right)\right|>w / \log \Delta \Longrightarrow \sum_{e^{\prime} \in C\left(e_{j}\right)}\left|s\left(e^{\prime}\right)\right|>w / \log \Delta
$$

Dividing both sides by $w$, we get $\sum_{e^{\prime} \in C\left(e_{j}\right)} x\left(e^{\prime}\right)>$ $1 / \log \Delta$, which contradicts the condition on $\bar{x}$.

In the following Lemma we prove that Algorithm 1, produces a valid schedule.

Lemma 5: Let $\bar{x}$ be a feasible solution to the program $\mathcal{P}\left(1 /(1+\log \Delta), \mathcal{I}^{\prime}=(V, E, \mathcal{D}, J /(1+\epsilon))\right)$, for a constant $\epsilon>0$. Then, Algorithm FrameSchedule produces a valid schedule corresponding to $\bar{x}$ for the instance $\mathcal{I}=(V, E, \mathcal{D}, J)$, of TM-SINR in which the SINR constraints are satisfied at all receivers, for constants $a$ and $\alpha$ defined in Section IV-C.

Proof: We show that at any time $t$, the set $E_{t}$ of links scheduled at this time in $\mathcal{S}$ can indeed be transmitted simultaneously, while satisfying the SINR constraints at each receiver, in the instance $\mathcal{I}$. Let $E_{t}=\left\{e_{j}=\left(u_{j}, v_{j}\right): j=1, \ldots, s\right\}$.

By construction, there exists a set $B_{i}$ such that $E_{t} \subseteq B_{i}$. Consider two edges $e_{j}, e_{m} \in E_{t}$ with $\ell\left(e_{j}\right) \leq \ell\left(e_{m}\right)$. Since these two edges are scheduled simultaneously, it must be the case that $e_{m} \notin C\left(e_{j}\right)$, which implies $d\left(u_{j}, u_{m}\right)>$ $a \max \left\{\ell\left(e_{j}\right), \ell\left(e_{m}\right)\right\}$. For any $e_{j} \in B_{i}$, we have $\ell\left(e_{j}\right) \in$ $\left[2^{i}, 2^{i+1}\right)$, and so $a 2^{i} \geq a \ell\left(e_{j}\right) / 2$. This implies that if we place a disk of radius $a \ell\left(e_{j}\right) / 4$ centered at the end points of each edge in $E_{t}$, all these disks would be disjoint.

Consider any $e_{j}=\left(u_{j}, v_{j}\right) \in E_{t}$. We estimate the SINR at $v_{j}$ in the following manner. As in [6], [18], we partition the plane into rings $R_{d}$ centered at $u_{j}$ (cf. Figure 2) for $d=0,1, \ldots$, each of width $a \ell\left(e_{j}\right)$ around $u_{j}$. Each ring $R_{d}$ consists of all links $e_{m}=\left(u_{m}, v_{m}\right)$, for which $d a \ell\left(e_{j}\right) \leq d\left(u_{j}, u_{m}\right)<(d+1) a \ell\left(e_{j}\right)$. As derived earlier, for any $e_{m} \neq e_{j}$, we have $d\left(u_{j}, u_{m}\right)>a \max \left\{\ell\left(e_{j}\right), \ell\left(e_{m}\right)\right\}$, which implies $R_{0}$ does not contain any links in $E_{t}$ other than $e_{j}$. By definition, the area of $R_{d}$ is $\pi\left[\left((d+1) a \ell\left(e_{j}\right)\right)^{2}-\right.$ 


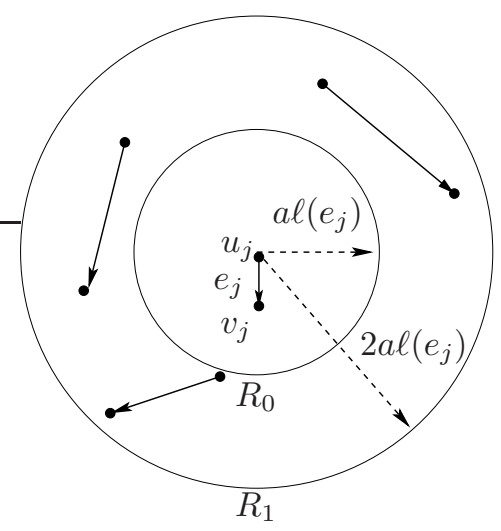

Fig. 2. For a given link $e_{j}=\left(u_{j}, v_{j}\right) \in E_{t}$, construct rings of radius $a \ell\left(e_{j}\right)$ around $u_{j}$. We calculate the interference experienced by node $v_{j}$ due to other simultaneously transmitting links.

$\left.\left(d a \ell\left(e_{j}\right)\right)^{2}\right]=\pi a^{2}(2 d+1) \ell\left(e_{j}\right)^{2} \leq 3 \pi d a^{2} \ell\left(e_{j}\right)^{2}$, and so the non-overlapping disks property implies that the number of transmitters in $R_{d}$ is at most

$$
\frac{3 \pi d a^{2} \ell\left(e_{j}\right)^{2}}{\pi a^{2} \ell\left(e_{j}\right)^{2} / 16} \leq 48 d
$$

Next, that for each $e_{m} \in R_{d}$, we have $d\left(u_{m}, v_{j}\right) \geq(a d-$ 1) $\ell\left(e_{j}\right) \geq \frac{a d}{2} \ell\left(e_{j}\right)$, since $a \geq 2$. Therefore, the interference at $v_{j}$ due to nodes in $R_{d}$, denoted by $\mathcal{I}_{d}\left(v_{j}\right)$, is bounded as follows,

$$
\mathcal{I}_{d}\left(v_{j}\right) \leq 48 \cdot d \cdot 2^{\alpha} \frac{J}{\left(\operatorname{ad\ell }\left(e_{j}\right)\right)^{\alpha}}=2^{\alpha} \frac{48 J}{a^{\alpha} d^{\alpha-1} \ell\left(e_{j}\right)^{\alpha}}
$$

Summing up the interference over all rings $R_{d}$, we have,

$$
\begin{aligned}
\sum_{d=1}^{\infty} \mathcal{I}_{d}\left(v_{j}\right) & \leq 2^{\alpha} \frac{48 J}{a^{\alpha} \ell\left(e_{j}\right)^{\alpha}} \sum_{d=1}^{\infty} \frac{1}{d^{\alpha-1}} \\
& \leq 2^{\alpha} \frac{48 J}{a^{\alpha} \ell\left(e_{j}\right)^{\alpha}} \int_{1}^{\infty} \frac{d x}{x^{\alpha-1}} \\
& \leq \frac{2^{\alpha} 48 J}{a^{\alpha} \ell\left(e_{j}\right)^{\alpha}(\alpha-2)} .
\end{aligned}
$$

Therefore the SINR at receiver $v_{j}$ is at least

$\frac{J}{\ell\left(e_{j}\right)^{\alpha}\left[N_{0}+\frac{2^{\alpha} 48 J}{a^{\alpha} \ell\left(e_{j}\right)^{\alpha}(\alpha-2)}\right]} \geq \frac{J}{\ell\left(e_{j}\right)^{\alpha}\left[\frac{J}{(1+\epsilon) \beta \ell\left(e_{j}\right)^{\alpha}}+\frac{\epsilon J}{(1+\epsilon) \beta \ell\left(e_{j}\right)^{\alpha}}\right]}$

which is at least $\beta$ if $a^{\alpha} \geq 2^{\alpha} \frac{48 \beta(\epsilon+1)}{\epsilon(\alpha-2)}$. Note that we have used the fact that $J \geq(1+\epsilon) \beta N_{0} \ell\left(e_{j}\right)^{\alpha}$, since we are analyzing the feasibility of the schedule $\mathcal{S}$ for the instance $\mathcal{I}$ of TM-SINR.

It is crucial to note that Lemma 5 proves that the schedule corresponding to the vector $\bar{x}$ produced by Algorithm FrameSchedule is valid not for the original instance $\mathcal{I}^{\prime}=$ $(V, E, \mathcal{D}, J /(1+\epsilon))$ of TM-SINR, but for a different instance $\mathcal{I}=(V, E, \mathcal{D}, J)$, so that for all $e \in E, J \geq(1+\epsilon) \beta N_{0} \ell(e)^{\alpha}$.

\section{Putting everything together}

For an input instance $\mathcal{I}=(V, E, \mathcal{D}, J)$ of TM-SINR, our algorithm computes the optimum solution $\bar{x}$ to the linear program $\mathcal{P}\left(1 /(1+\log \Delta), \mathcal{I}^{\prime}=\left(V, E^{\prime}, \mathcal{D}, J /(1+\epsilon)\right)\right)$, where $E^{\prime}=\left\{e \in E: J \geq(1+\epsilon) \beta N_{0} \ell(e)^{\alpha}\right\}$. From Lemma 5, it follows that $\bar{x}$ can be scheduled feasibly for the instance $\mathcal{I}$. The following theorem shows that the rate achieved by $\bar{x}$ is within a provable factor of $r_{o p t}\left(\mathcal{I}^{\prime}\right)$ - thus, this is a bicriteria approximation, in which we compare the quality of the solution produced by our algorithm with respect to the optimum for an instance that uses slightly less power.

Theorem 1: As defined above, let $\mathcal{I}=(V, E, \mathcal{D}, J)$ be an instance of TM-SINR with uniform power level $J$, and let $\mathcal{I}^{\prime}=\left(V, E^{\prime}, \mathcal{D}, J /(1+\epsilon)\right)$ be the corresponding instance, defined by using power levels $J /(1+\epsilon)$, with $E^{\prime}=\{e \in$ $\left.E: J \geq(1+\epsilon) \beta N_{0} \ell(e)^{\alpha}\right\}$, for any $\epsilon>0$. The optimum solution $\bar{x}$ to the program $\mathcal{P}\left(1 /(1+\log \Delta), \mathcal{I}^{\prime}\right)$ is a feasible and stable link utilization vector for the instance $\mathcal{I}$, and results in a total throughput of at least $\Omega\left(r_{\text {opt }}\left(\mathcal{I}^{\prime}\right) /(1+\log \Delta)\right)$.

Proof: Let $\bar{x}_{\text {opt }}$ be the optimum utilization vector for the instance $\mathcal{I}^{\prime}$ of TM-SINR, achieving a total throughput rate of $r_{\text {opt }}\left(\mathcal{I}^{\prime}\right)$. Note Lemma 3 holds for both the instances $\mathcal{I}$ and $\mathcal{I}^{\prime}$. From Lemma 3 , it follows that $\bar{x}_{\text {opt }}$ is feasible solution to the program $\mathcal{P}\left(\lambda_{0}, \mathcal{I}^{\prime}\right)$, for the constant $\lambda_{0}$ defined in Lemma 3. Since $\mathcal{P}\left(\lambda_{0}, \mathcal{I}^{\prime}\right)$ is a linear program, it follows that the utilization vector $\bar{y}=\frac{1}{\lambda_{0} \log \Delta} \bar{x}_{o p t}$ is a feasible solution to the program $\mathcal{P}\left(1 /(1+\log \Delta), \mathcal{I}^{\prime}\right)$, and results in a total throughput rate of $\frac{r_{o p t}\left(\mathcal{I}^{\prime}\right)}{\lambda_{0} \log \Delta}$. This implies that the optimum solution $\bar{x}$ to the program $\mathcal{P}\left(1 /(1+\log \Delta), \mathcal{I}^{\prime}\right)$ also results in a total throughput rate of at least $\frac{r_{o p t}\left(\mathcal{I}^{\prime}\right)}{\lambda_{0} \log \Delta}$. Finally, by Lemma 5, it follows that $\bar{x}$ can be scheduled feasibly for the instance $\mathcal{I}$ of TM-SINR. Therefore, the theorem follows.

\section{THROUGHPUT MAXIMIZATION FOR NON-UNIFORM POWER LEVELS}

In the previous section, we assumed that the power vector $\bar{J}$ was uniform with $J(e)=J$ for each edge $e$. We now extend this to the non-uniform setting where $J(e)$ need not be the same for every edge $e$. Let $\bar{J}$ be the corresponding power level vector.

\section{A. Problem Formulation}

The problem formulation for the TM-SINR problem for non-uniform power levels is similar to the one presented in Section VI-A. Recall the notation defined in Section IV. We partition the set $E$ of edges into sets $H_{k}^{i}=\{e=$ $\left.(u, v) \in E: \ell(e) \in\left[2^{i}, 2^{i+1}\right), J(e) \in\left[2^{k}, 2^{k+1}\right)\right\}, \forall i \in$ $\{0, \ldots,(\log \Delta-1)\}, k \in\{0, \ldots,(\log \Gamma-1)\}$. For an instance $\mathcal{I}=(V, E, \mathcal{D}, \bar{J})$ of TM-SINR, we define a different formulation $\mathcal{P}_{n}(\lambda, \mathcal{I})$ by replacing the constraints (5) in the program $\mathcal{P}(\lambda, \mathcal{I})$ by the constraints

$$
x(e)+\sum_{e^{\prime} \in C(e) \cap H_{k}^{i}} x\left(e^{\prime}\right) \leq \lambda,
$$

for all $i \in\{0, \ldots,(\log \Delta-1)\}, k \in\{0, \ldots,(\log \Gamma-1)\}$, and for all $e \in H_{k}^{i}$. 


\section{B. Link-Flow Scheduling: Necessary Conditions}

Lemma 6: Let $\bar{x}$ be any feasible link utilization vector for the instance $\mathcal{I}=(V, E, \mathcal{D}, \bar{J})$ of TM-SINR. Then, $\bar{x}$ is a feasible solution to the program $\mathcal{P}_{n}\left(\lambda_{1}, \mathcal{I}\right)$, where $\lambda_{1}=2 \frac{(2 a+1)^{\alpha}}{\beta}+1$, for constant $a$ defined in Section IV-C.

Proof: (Sketch) We mimic the proof of Lemma 3. Following the notation of Lemma 3 , we consider set $A_{t}$ to be the set of links that are scheduled at time $t$ in the feasible schedule corresponding to $\bar{x}$. Define $G_{i}=\left\{e \in E: 2^{i} \leq J(e)<\right.$ $\left.2^{i+1}\right\}$. Let $A_{t} \cap G_{i}=\left\{e_{i}=\left(u_{i}, v_{i}\right): i=1, \ldots, c\right\}$, with the edges numbered in non-decreasing order of their lengths. As in the proof of Lemma 3, by computing the SINR at the receiver $v_{c}$, we argue that at most $\lambda_{1}$ other transmissions in $A_{t} \cap G_{i}$ are simultaneously possible. Though the power levels are not uniform, considering the edges in $A_{t} \cap G_{i}$ implies that for any $e_{c}, e_{j} \in A_{t} \cap G_{i}$, we have $J\left(e_{c}\right) / 2 \leq J\left(e_{j}\right) \leq 2 J\left(e_{c}\right)$, which allows us to do the argument, with the constant $\lambda_{1}$ instead of $\lambda_{0}$.

We now consider the sufficient conditions for link-flow stability. Algorithm NonUniformFrameSchedule is the modified scheduling algorithm for this setting.

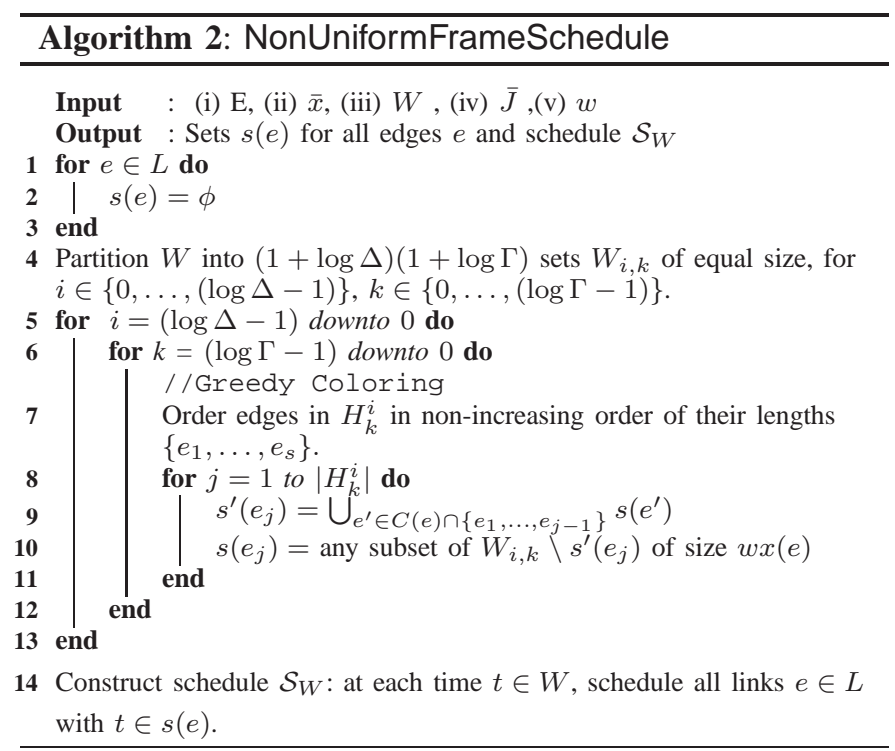

We construct a period schedule $\mathcal{S}$ using Algorithm 2 by repeating the schedule $\mathcal{S}_{W}$ for each frame $W$. It is easy to see that by making a minor modification to Lemma 4, it follows that this algorithm indeed assigns $x(e) w$ slots for each edge $e$. We now derive the conditions under which the schedule is valid.

Lemma 7: Let $\bar{x}$ be a feasible solution to the program $\mathcal{P}\left(1 /(1+\log \Delta)(1+\log \Gamma), \mathcal{I}^{\prime}=(V, E, \mathcal{D}, \bar{J} /(1+\epsilon))\right)$, for a constant $\epsilon>0$. Then, Algorithm NonUniformFrameSchedule produces a valid schedule corresponding to $\bar{x}$ for the instance $\mathcal{I}=(V, E, \mathcal{D}, \bar{J})$, of TM-SINR in which the SINR constraints are satisfied at all receivers, for constants $a$ and $\alpha$ defined in Section IV-C.
Proof: (Sketch) Consider any time slot $t$. Let $E_{t}$ denote the set of links scheduled in $\mathcal{S}$ at that time $t$. There exists a set $H_{k}^{i}$ for which $E_{t} \subseteq H_{k}^{i}$, for some $i \in\{0, \ldots,(\log \Delta-$ $1)\}, k \in\{0, \ldots,(\log \Gamma-1)\}$. Using the same argument and notation as in Lemma 5, we can see that for any edge $e_{j}=\left(u_{j}, v_{j}\right) \in E_{t}$, disks of radius $\frac{a \ell\left(e_{j}\right)}{4}$ centered at each node $e_{m}=\left(u_{m}, v_{m}\right) \in E_{t}$ are disjoint. Also since for any links $e_{m}=\left(u_{m}, v_{m}\right), e_{j}=\left(u_{j}, v_{j}\right) \in E_{t} \subseteq H_{k}^{i}$, $J\left(e_{m}\right) \in\left[2^{k}, 2^{k+1}\right)$ implies that $J\left(e_{m}\right) \leq 2 J\left(e_{j}\right)$. The rest of the proof remains similar to the proof of Lemma 5.

Theorem 2: Let $\bar{J}$ be a vector of non-uniform power levels, let $\mathcal{I}=(V, E, \mathcal{D}, \bar{J})$ be an instance of TM-SINR with nonlinear power levels, and let $\mathcal{I}^{\prime}=\left(V, E^{\prime}, \mathcal{D}, \frac{\bar{J}}{(1+\epsilon)}\right)$ be the corresponding instance obtained by using power levels $\frac{\bar{J}}{(1+\epsilon)}$, with $E^{\prime}=\left\{e \in E: J(e) \geq(1+\epsilon) \beta N_{0} \ell(e)^{\alpha}\right\}$, for any $\epsilon>0$. The optimum solution $\bar{x}$ to the program $\mathcal{P}\left(1 /(1+\log \Delta)(1+\log \Gamma), \mathcal{I}^{\prime}\right)$ is a feasible and stable link utilization vector for the instance $\mathcal{I}$, and results in a total throughput of at least $\Omega\left(r_{\text {opt }}\left(\mathcal{I}^{\prime}\right) /(1+\log \Delta)(1+\log \Gamma)\right)$.

As in the case of Theorem 1 for uniform power levels, this result is a bi-criteria approximation, in which the throughput rate guaranteed by our algorithm is compared to the optimum rate possible if slightly lower power levels are used.

\section{IMPROVED APPROXIMATIONS: LINEAR POWER LEVELS}

We now consider a special case of non-uniform power levels, in which $J(e)=c_{1} \ell(e)^{\alpha}$ for a constant $c_{1}$ such that $c_{1} \geq \beta N_{0}$ - this is also called the linear power level. Theorem 2 implies an approximation of $O\left((1+\log \Delta)^{2}\right)$ for this case, since $\Gamma=\Delta$. In this section, we show that this bound can be improved to $O(1+\log \Delta)$.

Let $\bar{J}$ be the power value vector with $J(e)=c_{1} \ell(e)^{\alpha}$, as defined above. We consider the problem instance $\mathcal{I}=$ $(V, E, \mathcal{D}, \bar{J})$ of TM-SINR. We show that in this case the program $\mathcal{P}(\lambda, \mathcal{I})$ itself can be used with a slight modification instead of the program $\mathcal{P}_{n}(\lambda, \mathcal{I})$ to get the better approximation. Recall the definition of the sets $B_{i}$ from Section IV. We form a linear program $\mathcal{P}_{l}(\lambda, \mathcal{I})$ by replacing the constraints 5 in the program $\mathcal{P}(\lambda, \mathcal{I})$ with constraints $x(e)+\sum_{e^{\prime} \in C(e) \cap B_{i}} x\left(e^{\prime}\right) \leq$ $\lambda$ for all $e \in B_{i}, \forall i \in\{0, \ldots,(\log \Delta-1)\}$.

Lemma 8: Let $\bar{x}$ be any feasible rate vector for the problem $\mathcal{I}=(V, E, \mathcal{D}, \bar{J})$. Then, $\bar{x}$ satisfies all the constraints of the program $\mathcal{P}\left(\lambda_{2}, \mathcal{I}\right)$, where $\lambda_{2}=2^{\alpha} \frac{(2 a+1)^{\alpha}}{\beta}+1$, for constant $a$ defined in Section IV-C.

Proof: (Sketch) We mimic the proof of Lemma 3. Recall the notation of set $A_{t}$ from Lemma 3. We show that number of links simultaneously scheduled from set $A_{t} \cap B_{i}, \forall e \in E, \forall i \in$ $\{0, \ldots,(\log \Delta-1)\}$ is at most $\lambda_{2}$. Again, we consider the SINR at the receiver of the longest edge $e_{c} \in A_{t} \cap B_{i}$. Note that $\forall e_{j}, e_{c} \in A_{t} \cap B_{i}$, we have $J\left(e_{c}\right) / 2^{\alpha} \leq J\left(e_{j}\right) \leq 2^{\alpha} J\left(e_{c}\right)$. Following the same sequence of arguments as in Lemma 3, we can derive 


$$
\frac{J\left(e_{c}\right)}{d\left(u_{c}, v_{c}\right)^{\alpha}} \geq \beta N_{0}+\frac{\beta J\left(e_{c}\right)(c-1)}{2^{\alpha}(2 a+1)^{\alpha} d\left(u_{c}, v_{c}\right)^{\alpha}}
$$

which implies the bound on $\mid A_{t} \cap B_{i} \cap C\left(e_{c}\right)$.

Theorem 3: Let $\mathcal{I}=(V, E, \mathcal{D}, \bar{J})$ be an instance of TMSINR with linear power level, i.e., $J(e)=c_{1} \ell(e)^{\alpha}$ for all $e \in E$. Let $\mathcal{I}^{\prime}=\left(V, E^{\prime}, \mathcal{D}, \frac{\bar{J}}{(1+\epsilon)}\right)$ be the corresponding instance obtained by using power levels $\frac{\bar{J}}{(1+\epsilon)}$, for any $\epsilon>$ 0 , with $E^{\prime}=\left\{e \in E: J(e) \geq(1+\epsilon) c_{1} \ell(e)^{\alpha}\right\}$. The optimum solution $\bar{x}$ to the program $\mathcal{P}\left(1 /(1+\log \Delta), \mathcal{I}^{\prime}\right)$ is a feasible and stable link utilization vector for the instance $\mathcal{I}$, and results in a total throughput of at least $\Omega\left(r_{\text {opt }}\left(\mathcal{I}^{\prime}\right) /(1+\log \Delta)\right)$.

Proof: (Sketch) The proof of this lemma is similar to that of Lemmas 5, 4 and Theorem 1. We use Algorithm FrameSchedule to schedule the vector $\bar{x}$. Using similar techniques from Lemma 5, we show that the resulting schedule is valid. From the notation used in the proof of Lemma 5, recall the definition of $E_{t}$. We fix an edge $e_{j}=\left(u_{j}, v_{j}\right) \in E_{t}$. Using similar arguments we obtain the non-overlapping disk property. Further, for any two edge $e_{j}, e_{m} \in E_{t}$, we have $J\left(e_{m}\right) \leq 2^{\alpha} J\left(u_{j}\right)$. Following the same sequence of arguments, we partition the plane into rings $R_{d}$. The interference at node $v_{j}$ due to simultaneous transmissions the nodes in the ring $R_{d}$ can be obtained as,

$$
\mathcal{I}_{d}\left(v_{j}\right) \leq 2^{2 \alpha} \frac{48\left(e_{j}\right)}{a^{\alpha} d^{\alpha-1} \ell\left(e_{j}\right)^{\alpha}}
$$

It is easy to verify that the remaining steps follow, with the above modification to the expression for $\mathcal{I}_{d}\left(v_{j}\right)$ and therefore the theorem follows.

\section{CONCLUSION}

We study the problem of throughput maximization in arbitrary wireless networks with SINR constraints problem from a theoretical perspective, and take the first steps towards developing efficient algorithms for this problem. Our results show that the comparison between SINR and graph-based models is complicated, and for different instances, different models might give higher estimates of the throughput capacity, suggesting the need for greater care in using these models. We develop the first provable algorithms for approximating the throughput capacity in the SINR models by means of a linear programming formulation, extending the recent work of [6], [13].

Extending these results to distributed algorithms would make them more useful from a practical point of view. This paper does not consider power control, and studying the problem of joint power control and throughput maximization would be an interesting extension. Extending these results to the generalized SINR models would also be an interesting problem.

Acknowledgments. The research of D. Chafekar, V.S.Anil
Kumar, and M.V. Marathe was supported in part by NSF Award CNS-0626964. A. Srinivasan was supported in part by NSF ITR Award CNS-0426683 and NSF Award CNS-0626964; part of this work was done while he was on sabbatical at the Network Dynamics and Simulation Science Laboratory of the Virginia Bioinformatics Institute, Virginia Tech.

\section{REFERENCES}

[1] A. Agarwal, and P. Kumar, Capacity bounds for ad hoc and hybrid wireless networks, in ACM SIGCOMM, pp. 71-81,July 2004.

[2] M. Alicherry, and R. Bhatia, Joint channel assignment and routing for throughput optimization in multi-radio wireless mesh networks, in ACM MOBICOM, pp. 58-72, August 2005.

[3] N. Bansal and Z. Liu. Capacity, delay and mobility in wireless ad-hoc networks, IEEE INFOCOM, April 2003.

[4] R. Bhatia, and M. Kodialam, On Power Efficient Communication over Multi-hop Wireless Networks: Joint Routing, Scheduling and Power Control, in IEEE INFOCOM, vol. 2, pp. 1457- 1466, March 2004

[5] C. Buragohain, S. Suri, C. Toth, and Y. Zhou, Improved Throughput Bounds for Interference-aware Routing in Wireless Networks, The 13th Annual International Computing and Combinatorics Conference, July 2007.

[6] D. Chafekar, V.S. Anil Kumar, M. Marathe, S. Parthasarathy, A. Srinivasan, and A. Srinivasan, Cross-Layer Latency Minimization in Wireless Networks with SINR Constraints, ACM MOBIHOC, pp. 110-119, September 2007.

[7] O. Goussevskaia, Y. Oswald, and R. Wattenhofer, Complexity in Geometric SINR, ACM MOBIHOC, pp. 100-109, September 2007.

[8] P. Gupta, and P. Kumar, The Capacity of Wireless Networks, IEEE Transactions on Information Theory, vol. 46, Issue 2, pp. 388-404, March 2000.

[9] K. Jain, J. Padhye, V. Padmanabhan, and L. Qiu, Impact of interference on multi-hop wireless network performance, ACM MOBICOM, pp. 6680, 2003

[10] M. Kodialam and T. Nandagopal, Characterizing Achievable Rates in Multi-hop Wireless Networks: The Joint Routing and Scheduling Problem, in ACM MOBICOM, pp. 42 - 54, September 2003.

[11] M. Kodialam and T. Nandagopal, Characterizing the Capacity Region in Multi-Radio Multi-Channel Wireless Mesh Networks, in ACM MOBICOM, pp. 73 - 87, August 2005.

[12] U. Kozat and L. Tassiulas. Throughput Capacity in Random Adhoc Networks with Infrastructure Support, Proc. 9th Annual ACM International Conference on Mobile computing and networking, Sept. 2003.

[13] V.S. Anil Kumar, M. Marathe, S. Parthasarathy, and A. Srinivasan, Algorithmic Aspects of Capacity in Wireless Networks, in ACM SIGMETRICS, pp. 133-144, 2005.

[14] X. Lin and N. Shroff, Joint Rate Control and Scheduling in Multihop Wireless Networks, in 43rd IEEE Conference on Decision and Control, Paradise Island, vol. 2, pp. 1484- 1489, December 2004.

[15] X. Lin and N. Shroff, The Impact of Imperfect Scheduling on Crosslayer Rate Control in Multihop Wireless Networks, in IEEE INFOCOM, vol. 3, pp. 1804- 1814, March 2005.

[16] T. Moscibroda, and R. Wattenhofer, The Complexity of Connectivity in Wireless Networks, IEEE INFOCOM, pp. 1-13, April 2006.

[17] T. Moscibroda, R. Wattenhofer, and A. Zollinger. Protocol Design Beyond Graph-Based Models, 5th Workshop on Hot Topics in Networks (HotNets), Irvine, California, USA, November 2006.

[18] T. Moscibroda, R. Wattenhofer, and A. Zollinger, Topology Control Meets SINR: The Scheduling Complexity of Arbitrary Topologies, ACM MOBIHOC, pp. 310-321, 2006.

[19] R. Ramanathan, A Unified Framework and Algorithm for (T/F/C) DMA Channel Assignment in Wireless Networks, ACM INFOCOM , pp. 900907, April 1997.

[20] G. Sharma, R. Mazumdar, N. Shroff, On the complexity of scheduling in wireless networks, ACM MOBICOM , pp. 227-238, 2006.

[21] S. Toumpis, and A. Goldsmith, Capacity Regions for Wireless Ad Hoc Networks, International Symposium on Communication Theory and Applications, April 2001. 